A pidologie, 1986, 17 (4), 279-306

\title{
APPORT DE LA PALYNOLOGIE A LA CONNAISSANCE DES RELATIONS ABEILLES/PLANTES EN SAVANES ARBORÉES DU TOGO ET DU BÉNIN
}

\author{
Danielle LOBREAU-CALLEN*, Roger DARCHEN * et Annick LE THOMAS *: \\ * Laboratoire de Taxsnomie et Ecologie des Flores Tropicales, C.N.R.S., \\ LA 218 et Laboratoire de Phytomorphologie de VE.P.H.E., \\ 16, rite Buffon, 75005 Paris, France \\ C.N.R.S., Station Biologique, 24620 Les Eyzies, France \\ :3aboratoire de Phytonorphologie de l'E.P.H.E., I6, rue Buffon, 75005 Paris, France
}

\section{RÉSUMÉ}

Une analyse pollinique a été effectuée sur 13 échantillons de miels provenant des régions du Togo et du Bénin où se pratiquent à la fois l'apiculture traditionnelle et l'apiculture par centrifugation; deux échantillons proviennent de ruches naturelles d'Hypotrigones. Cette analyse montre, que tout en reflétant la flore de ces régions en saison sèche, le spectre pollinique est très nettement appauvri Apis et Hypotrigona visitant un nombre limité de plantes fleuries à cette époque. Le choix restreint de ces abeilles implique plusieurs facteurs complémentaires : la proximité des plantes autour des ruchers traduisant la recherche d'une dépense énergétique minimale, leur production de nectar, leurs structures florales particulièrement attractives et, dans certains cas, la satisfaction de besoins accessoires pour les ruches. Le butinage intense de certaines plantes cheiroptérophiles (Parkia) ne semble pas pouvoir s'expliquer par une action pollinisatrice complémentaire mais plutôt par un comportement d'utilisatrices de gommes ou de résines.

\section{INTRODUCTION}

Le rôle des interrelations entre plantes at pollinisateurs dans la dynamique des écosystèmes a été très longtemps négligé, mais depuis quelques années, il est devenu un axe de recherche privilégié en biocoenologie. Les abeilles constituent, en particulier, un des principaux groupes d'insectes visiteurs de plantes. De nombreuses études ont été réalisées sur leur comportement en région tempérée, mais peu de travaux de ce genre existent pour les régions tropicales. On constate, en effet, que très peu d'inventaires des plantes mellifères africaines sont à notre disposition (SMITH, 1957, 1960; Crane et al., 1984), quelques-uns étant strictement régionaux : SowUmNi, 1976 pour le Nigeria, ou encore Lobriau- 
Callen, 1985 ; Lobrlau-Callen et Coutin, 1984 et sous presse, pour le Sénégal. Au Bénin et au Togo, aucune étude de cet ordre n'a été faite jusqu’à présent.

Les récoltes d'un certain nombre d'échantillons de miel provenant d'Apis mellifera et Hypotrigona sp. '1!, effectuées au cours d'une mission dans ces deux pays en 1984, nous ont incités à tenter d'analyser, par la mélisopalynologie, la stratégie de butinage de ces insectes et leur rôle dans la pollinisation de certaines plantes. C'est en effet une méthode globale qui permet, par un seul type d'analyse, celle du pollen extrait des miels, d'avoir une idée d'ensemble sur les rapports abeilles/plantes pour une période précise qui peut être assez longue.

Dans le cas des échantillons que nous analysons, cette méthode est entachée d'imperfection puisque la pollen n'a pu être extrait de miels directement récoltés dans les ruches, en raison du temps trop court passé sur le terrain. Il a été le plus souvent prélevé à partir de miels vendus au bord des routes et dans les villages; l'échantillonnage est donc imparfait, mais aucun doute ne subsiste en ce qui concerne l'origine de ces miels.

En dépit de ces réserves, cette analyse nous permet d'élargir les connaissances, encore trop sporadiques, sur la flore mellifère africaine et nous conduit à discerner certaines caractéristiques de la stratégie de butinage des abeilles sociales.

\section{MATÉRIEL ET MÉTHOdES}

Les récoltes de miels ont été effectuées entre le 2 et le 28 mars, c’est-à-dire à la fin de lat saison sèche, époque à laquelle les agriculteurs qui pratiquent une apiculture traditionnelle, font théoriquement leur unique récolte. Le tableau 1 rassemble les données concernant les lieux de provenance de ces miels ainsi que leur type d'extraction.

Les échantillons analysés proviennent de la région centrale du Togo, à Soutouboua et Sokodé. d'où la mission s'est poursuivie vers les secteurs agricoles de Tchaondjo, Tchoidé, Tabindé, Blitta, Lamatessi, Yara Kabyé, Kara et Warengo. Au Bénin, ils ont été collectés dans les savanes du Nord-Ouest ou du Sud-Ouest du Massif d'Atakora, voisines de Natitingou, à Tanguieta, Tanouyou, Boukoumbé et Peporyoyou, vers le Sud-Est, dans la région de Parakou et au Nord vers Kandi (fig. 1). L'échantillonnage est donc limité aux régions du Centre et Centre-Nord des deux pays, c'est-à-dire dans les régions de savanes boisées guinéennes, à l'exception d'un miel provenant de savanes boisées soudanaises $\left(\mathrm{n}^{\circ} 170\right)$.

Les analyses portent toutes, au moins sur $10 \mathrm{ml}$ de miels qui ont été traités selon la méthode de la Commission internationale de Botanique apicole décrite par Louveaux et al. (1970) et aménagée par divers auteurs (Gadbin, 1980; Lieux, 1980; Lobreau-Callen et Callen, 1983) afin d'éliminer les sucres, les cires et les protéines, les membranes pectocellulosiques,... Pour cela,

(1) Les difficultés d'identification des Hypotrigona africains n'ont pas permis de détermincr avec certitude l'espèce à laquelle appartiennent ces abeilles sociales. 


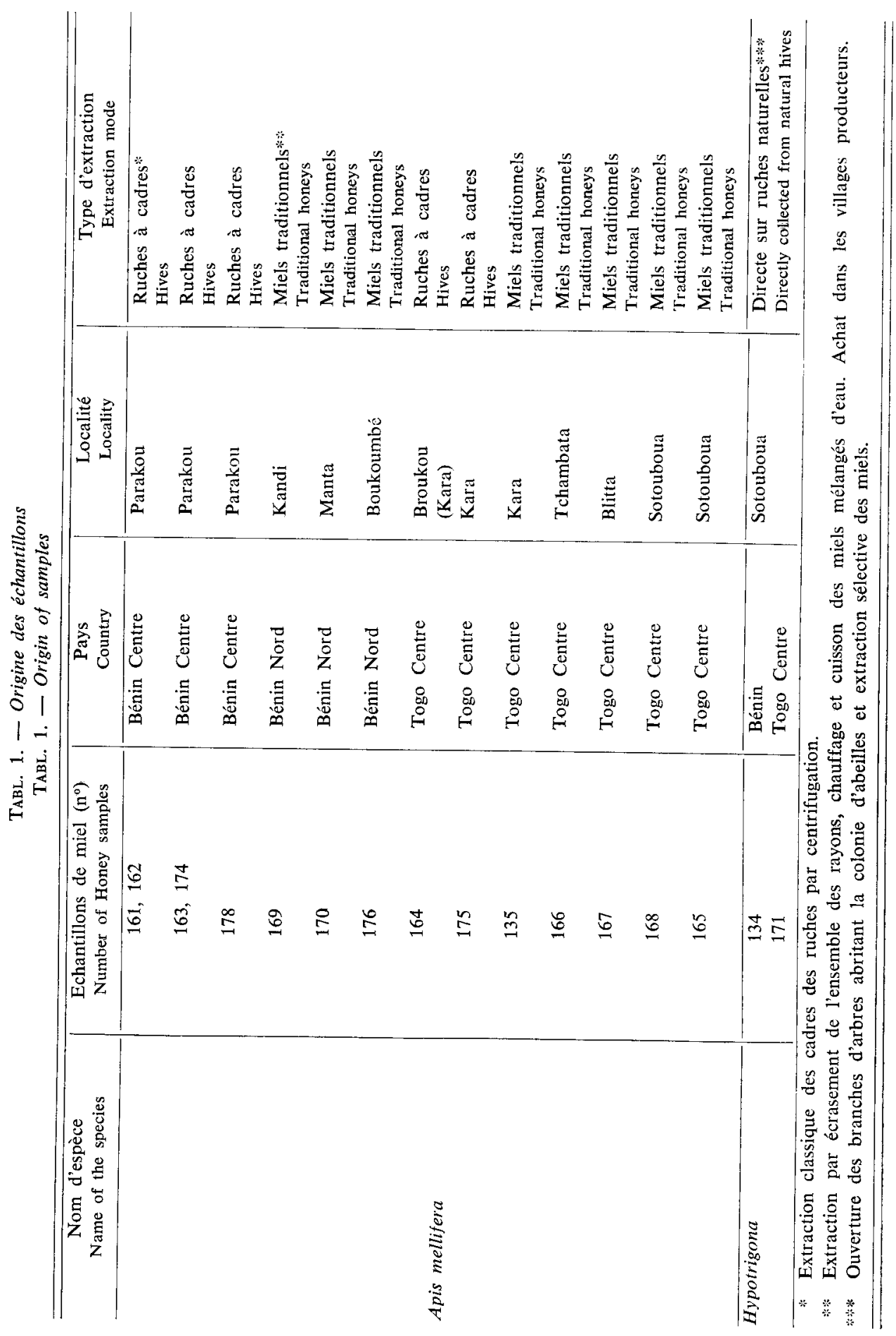




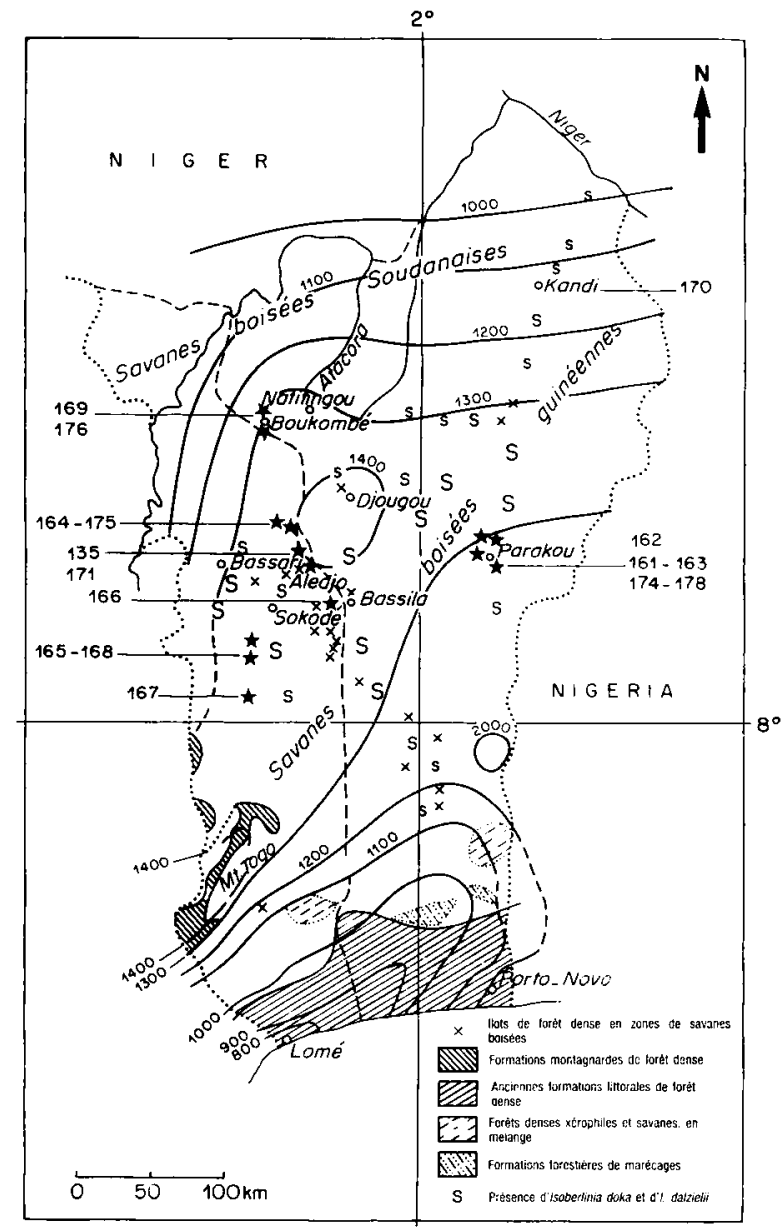

Fig. 1. - Localisation des échantillons de miel récoltés au Togo et au Bénin, en fonction des courbes pluviométriques et des formations forestières, d'après Aubréville, 1937

Fig. 1. - Localization of honey samples collected in Togo and Benin, with respect to pluviometric contours and forest communities, from Aubréville, 1937

les miels sont lessivés par des bains successifs d'eau chaude $\left(50^{\circ}\right.$ environ) acidulée avec quelques gouttes $\mathrm{d}^{\prime} \mathrm{H}_{2} \mathrm{SO}_{4}$, suivis d'autant de centrifugations. Ceux qui contiennent une imporante couche de "gel " sont traités avec un détergent puissant (Mucapur) des lipides, des résines naturelles, des mucilages ou des gommes, puis rincés à l'acide chlorhydrique dilué. Lorsque le culot est réduit à une poudre fine, il est traité à l'acide acétique pour éliminer l'eau, puis acétolysé.

L'ensemble des lames est alors exploré afin d'identifier les pollens représentés, puis des comptages sont effectués en prenant des lignes à la périphérie et au centre de la préparation, de telle sorte que les poliens comptés soient représentatifs de l'ensemble de la population palynologique et que toutes les espèces identifiées au préalable soient retrouvées.

On admet communément qu'avec un nombre moyen de 1200 pollens comptés, les espèces végétales les plus significatives sont recensées dans les miels européens, et que leur pourcentage est donc stable et fiable (Vergeron, 1964). Pour nos analyses, cette méthode de pourcentage stable des grains de pollens représentatifs sur une lame a été adoptée, ce qui entraîne une variation assez grande dans le nombre de pollens comptés par échantillon de miel en fonction de sa composition. 


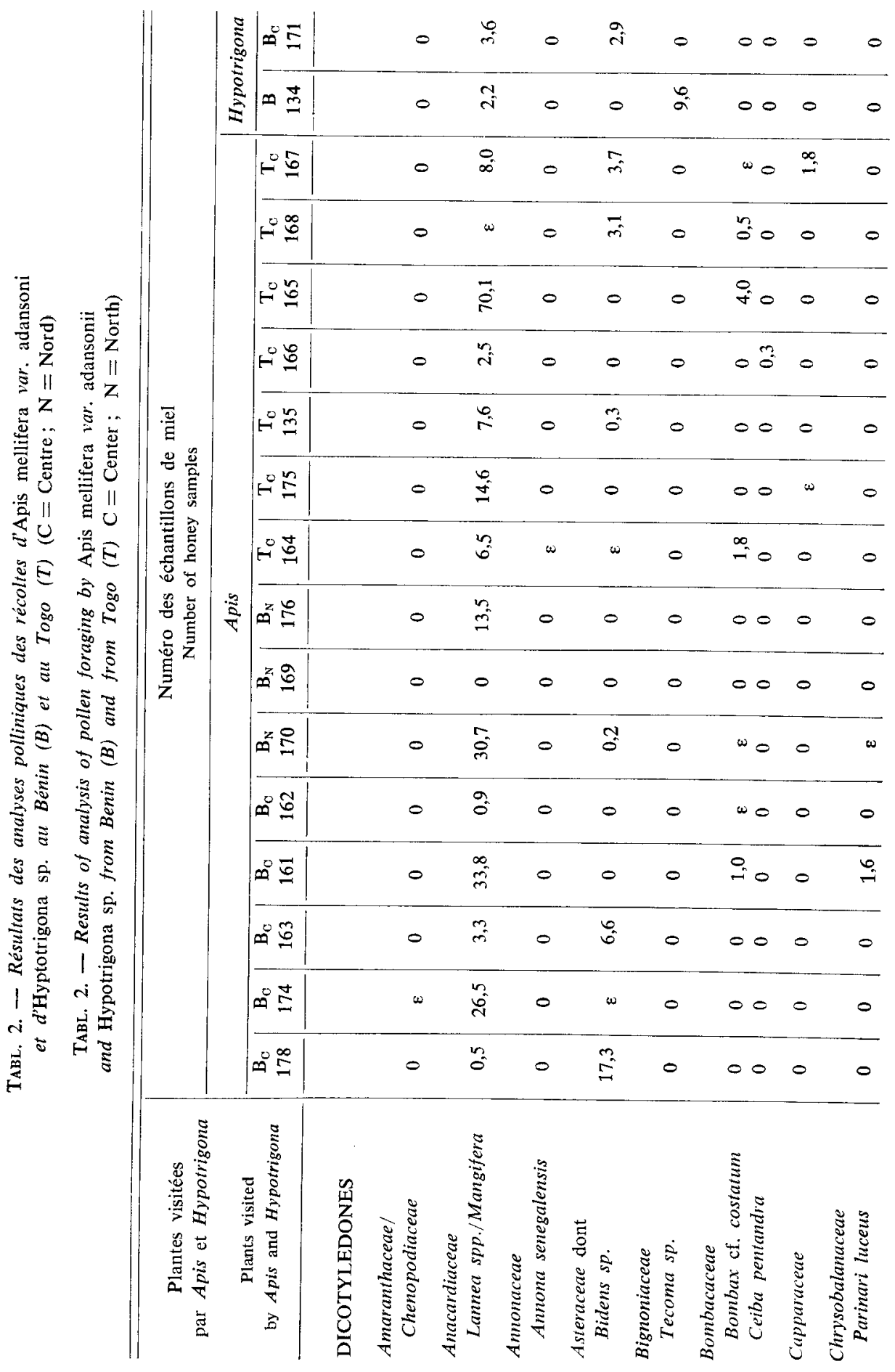




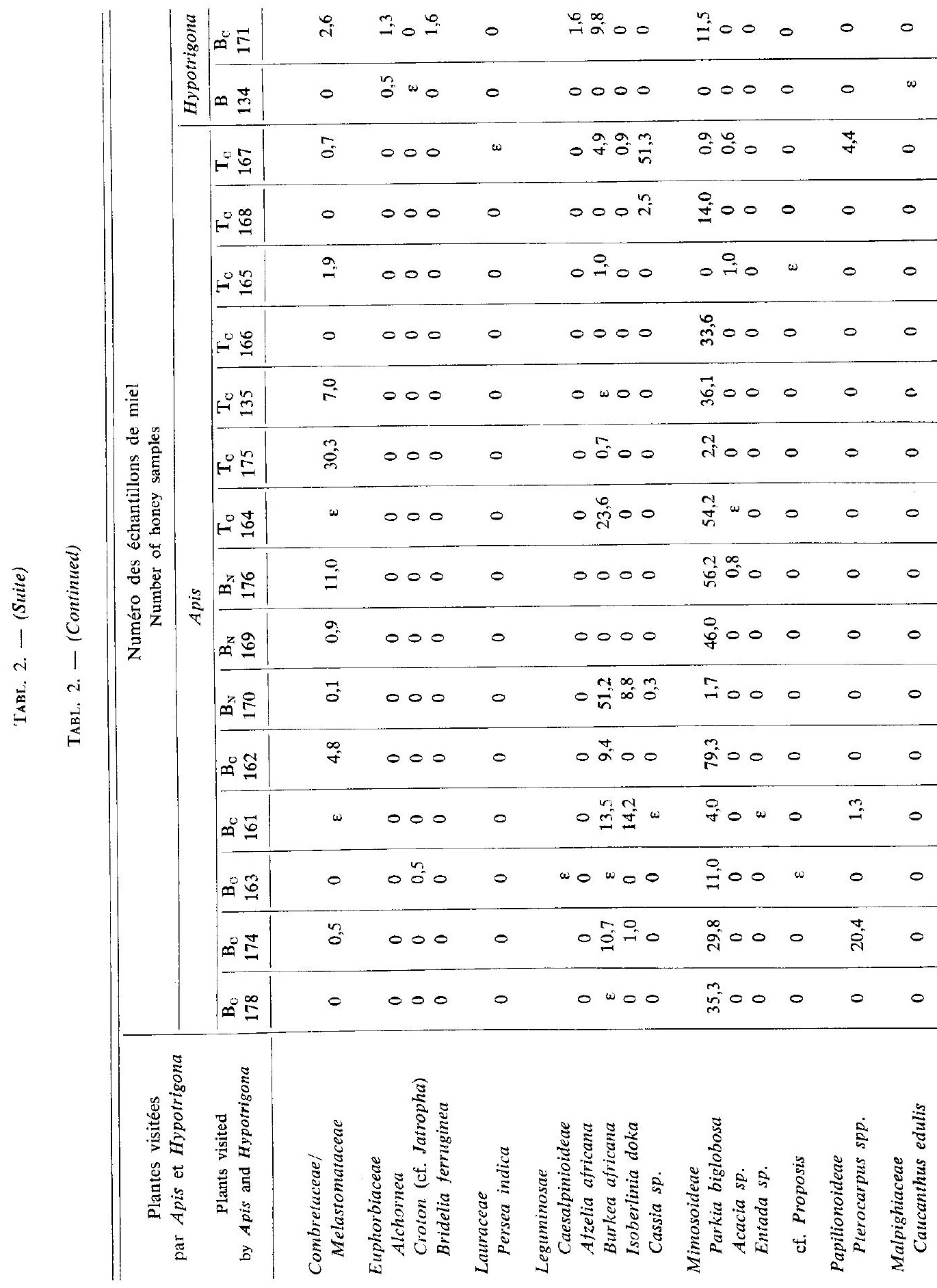




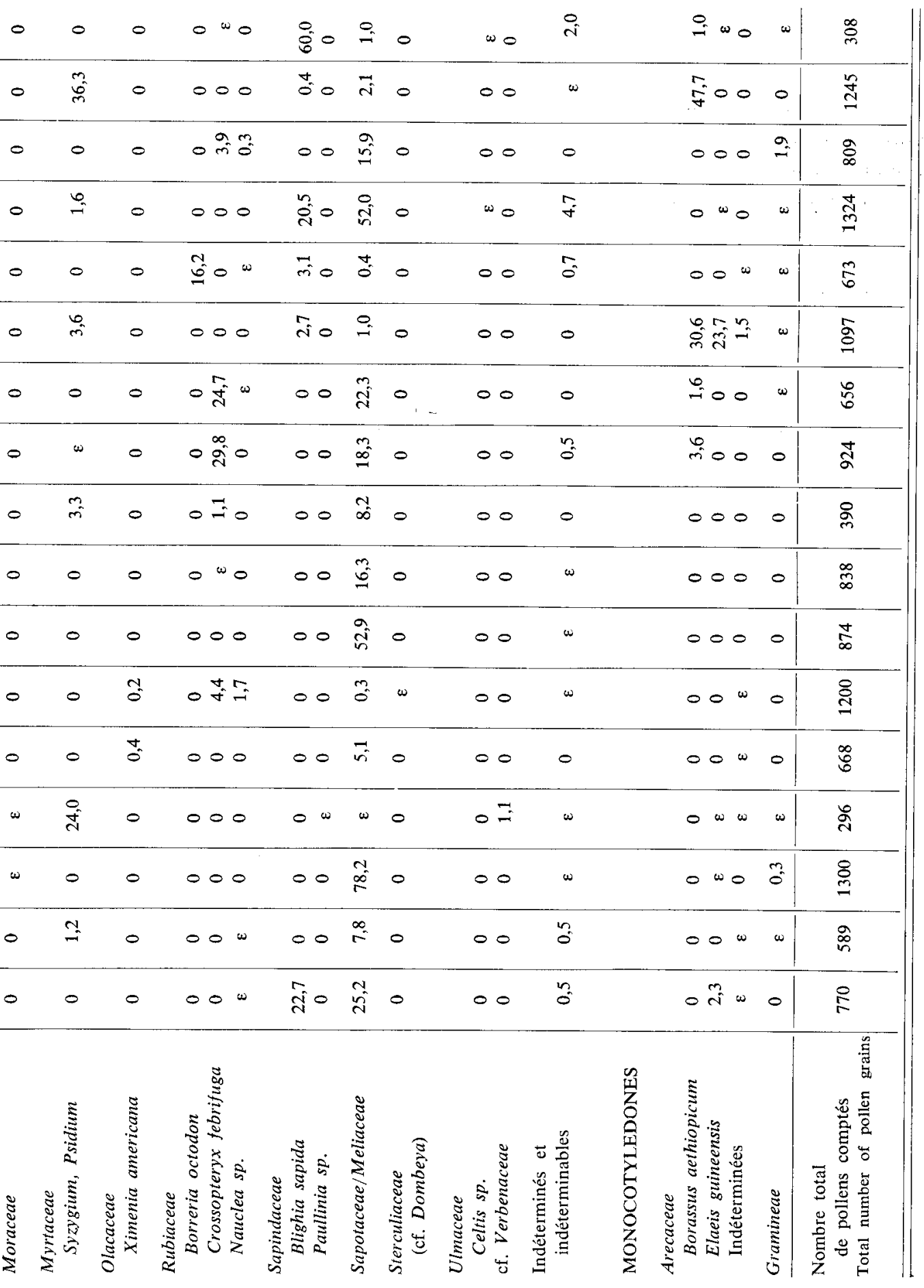




\section{RÉSULTATS}

Les résultats de nos analyses sont exposés dans deux tableaux complémentaires. Le tableau 2 donne, d'une part, le nombre total de grains de pollens comptés dans chaque échantillon de miel, d'autre part le pourcentage de pollens des différents taxons rencontrés et appartenant pour l'ensemble à une vingtaine de familles de Dicotylédones et Monocotylédones. Le total des pourcentages atteint très rarement 100 en raison des très faibles quantités de pollens $(\varepsilon)$ rencontrés pour certains taxons. Le tableau 3 distingue, dans les différentes familles observées, trois groupes de plantes suivant l'attirance qu'elles exercent sur Apis mellifera, compte tenu de la quantité de pollens et de nectar récoltée.

En outre, la comparaison avec les données bibliographiques a permis d'affiner certaines déterminations au niveau spécifique et de faire ressortir les espèces nouvellement signalées dans les miels africains. Ainsi, par exemple, la très grande ressemblance des pollens de Sapotaceae et de Meliaceae, n'a pas toujours permis de les identifier avec précision dans les comptages, ce qui nous a contraints à les réunir sous un même pourcentage (tabl. 2). Pourtant, si dans la majeure partie des cas, ils peuvent être rapportés à l'espèce Butyrospermum parkii (Sapotaceae), abondamment représentée dans les savanes étudiées, il n'est pas exclu qu'ils puissent également traduire la présence de Khaya senegalensis qui fait partie de la composition floristique de la région et est signalée comme espèce mellifère dans la littérature (Crane et al., 1984). Certains types polliniques extrêmes peuvent d'ailleurs lui être rapportés.

La présentation de ces tableaux est complétée par l'illustration du pollen des taxons les plus représentatifs provenant des différents miels étudiés (fig. 2 et 3 ).

Selon les échantillons de miel, on constate, en premier lieu, une grande variation dans la richesse pollinique de chacun d'entre eux (nombre total de grains de pollens comptés, tabl. 2). En dehors de la méthode de comptage qui a été adoptée, on peut s'interroger sur les raisons de ces écarts. Apparemment ils ne semblent absolument pas liés au type de miel étudié (ruches à cadres miels traditionnels ou extraction directe sur ruches naturelles), ni au genre d'abeille butinante (Apis ou Hypotrigona). La pauvreté absolue en pollens des échantillons 161,164 et 171 traduit certainement le fait que nous sommes en présence de miels de nectar. En ce qui concerne les autres échantillons, on constate que la présence importante de pollens de Parkia est généralement corrélative d'un moins grand nombre de pollens comptés. On peut concevoir dans ce cas que les très grosses polyades de Parkia, composées de 32 grains, limitent en quelque sorte le nombre de grains de pollens par rapport à l'espace disponible sur une lame. $\mathrm{Si}$, d'autre part, on examine les variations qui existent dans le nombre de pollens pour des 
TABL. 3. - Liste des espèces exploitées par Apis mellifera

TABL. 3. - Species visited by Apis mellifera

\begin{tabular}{|c|c|c|c|}
\hline $\begin{array}{l}\text { Quantité de pollen } \\
\text { et de nectar } \\
\text { Amounts of pollen } \\
\text { and nectar }\end{array}$ & $\begin{array}{l}\text { Noms d'espèces } \\
\text { Names of the species }\end{array}$ & $\begin{array}{l}\text { Familles } \\
\text { Families }\end{array}$ & $\begin{array}{c}\text { Déjà signalées } \\
\text { Previously } \\
\text { mentionned }\end{array}$ \\
\hline $\begin{array}{l}\text { Abondante } \\
\text { Abundant }\end{array}$ & Lannea spp. & Anacardiaceae & $\mathrm{X}$ \\
\hline $\begin{array}{l}\text { Abondante } \\
\text { A bundant }\end{array}$ & L. acida A. Rich. & & \\
\hline $\begin{array}{l}\text { Abondante } \\
\text { A bundant }\end{array}$ & L. barteri (Oliv.) Engl. & & \\
\hline $\begin{array}{l}\text { Abondante } \\
\text { Abundant }\end{array}$ & L. egregia Engl. & & \\
\hline $\begin{array}{l}\text { Abondante } \\
\text { Abundant }\end{array}$ & Khaya senegalensis (Desv.) Juss. & Meliaceae & $\mathrm{X}$ \\
\hline $\begin{array}{l}\text { Abondante } \\
\text { Abundant }\end{array}$ & Parkia biglobosa (Jacq.) Benth. & Lesuminosae & $\mathrm{x}$ \\
\hline $\begin{array}{l}\text { Abondante } \\
\text { Abundant }\end{array}$ & Butyrospermum parkii Kotschy & Saporteceres & \\
\hline $\begin{array}{l}\text { Moyenne } \\
\text { Medium }\end{array}$ & Borassus aethiopicum (Mart.) Mart. & Aracene & \\
\hline $\begin{array}{l}\text { Moyenne } \\
\text { Medium }\end{array}$ & Burkea africana Hook & Leguminosae & \\
\hline $\begin{array}{l}\text { Moyenne } \\
\text { Medium }\end{array}$ & $\begin{array}{l}\text { Combretum ghazalensis Engl. \& } \\
\text { Diels }\end{array}$ & Combretaceae & $\mathrm{X}$ \\
\hline $\begin{array}{l}\text { Peu abondante } \\
\text { Little }\end{array}$ & Elaeis guineensis Jacq. & Araceae & $\mathrm{x}$ \\
\hline $\begin{array}{l}\text { Peu abondante } \\
\text { Little }\end{array}$ & Isoberlinia doka Craib. \& Stapf. & Leguminosae & $\mathrm{x}$ \\
\hline $\begin{array}{l}\text { Peu abondante } \\
\text { Little }\end{array}$ & Psidium guajava $\mathrm{L}$. & Myrtaceae & $\mathrm{x}$ \\
\hline $\begin{array}{l}\text { Peu abondante } \\
\text { Little }\end{array}$ & Pterocarpus erinaceus Poir. & Leguminosae & $x$ \\
\hline $\begin{array}{l}\text { Peu abondante } \\
\text { Little }\end{array}$ & Crossopteryx febrifuga Benth. & Rubiaceas & \\
\hline $\begin{array}{l}\text { Peu abondante } \\
\text { Little }\end{array}$ & Blighia sapida Koenig. & Sapindaceae & \\
\hline
\end{tabular}



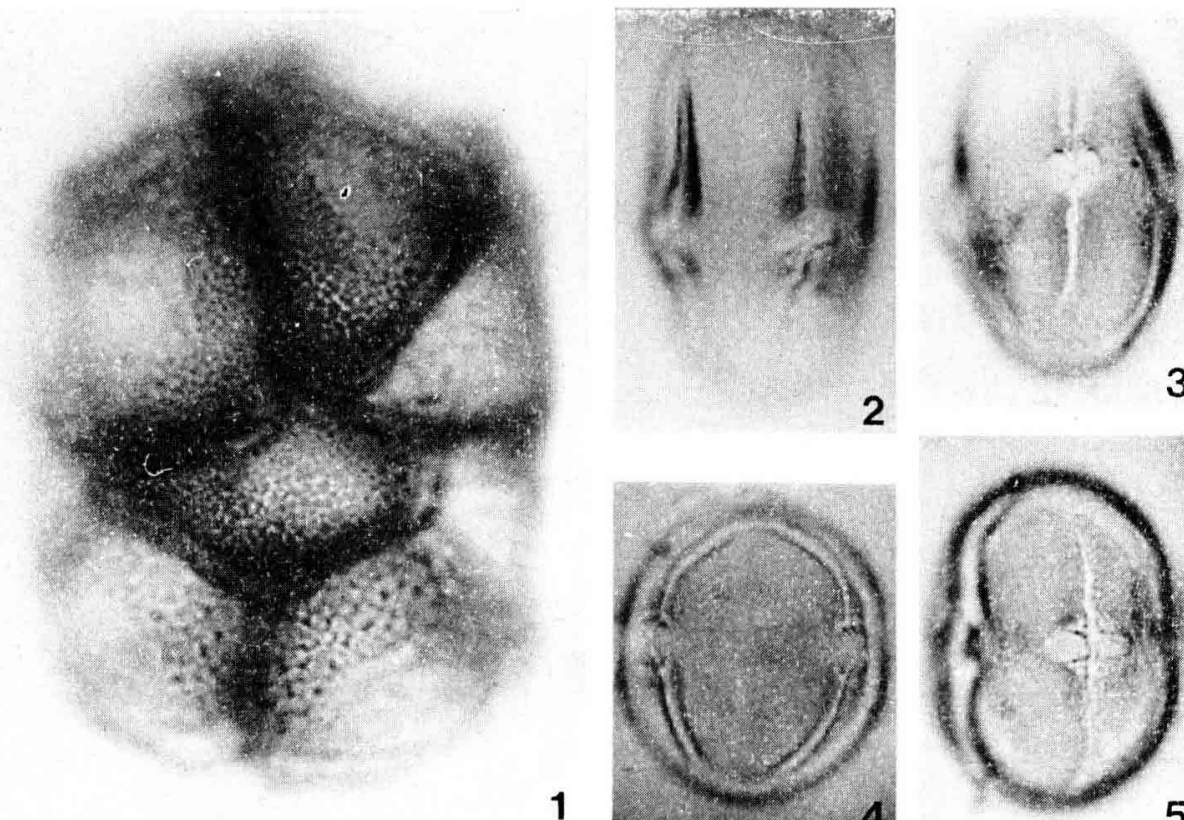

2

3
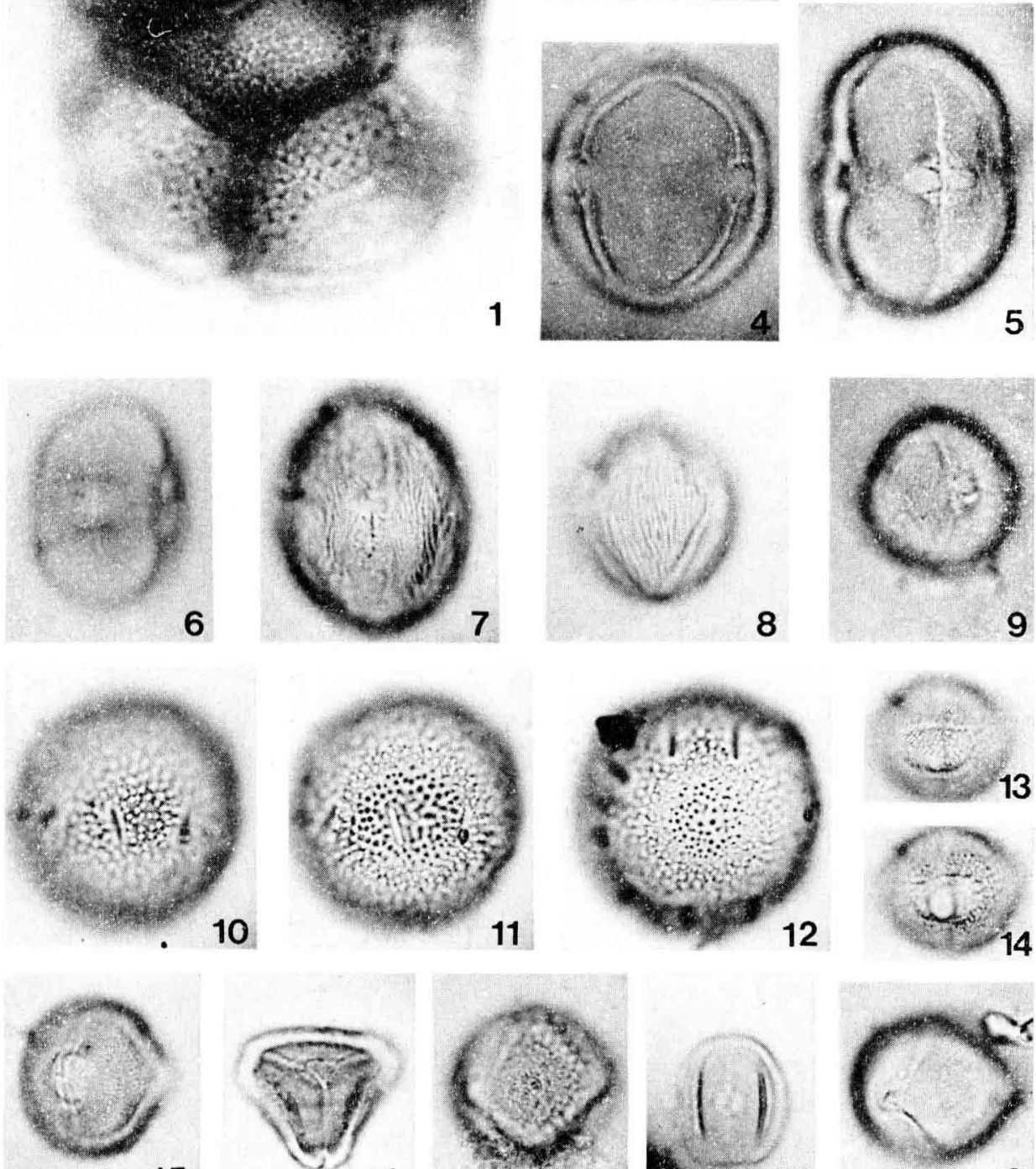

15
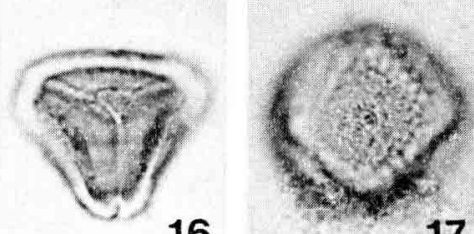

17.
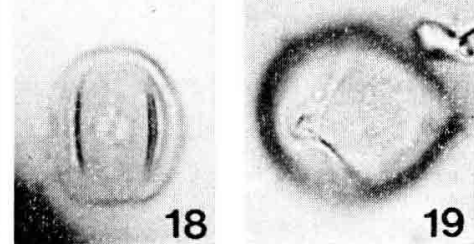

FIG. 2. - (Gr. $\times 1000)$. - 1 , Parkia biglobosa $\left(n^{\circ} 135\right) ; 2-3$, Butyrospermum parkii $\left(n^{\circ} 135\right)$; 4-5-6, Meliaceae-Sapotaceae [(4) $n^{\circ} 164$, (5) $\left.n^{0} 167,(6) n^{\circ} 135\right] ; 7-8$, Lannea sp. $\left(n^{\circ} 135\right)$;

9, Blighia sapida $\left(n^{0} 171\right) ; 10-12$, Borreria octodon ( $\left.n^{\circ} 164\right) ; 13-14$, Crossopteryx febrifuga $\left(n^{\circ} 135\right)$; 15 , Pterocarpus sp. $\left(n^{\circ} 174\right) ; 16$, Syzygium sp. $\left(n^{\circ} 134\right) ; 17$, Burkea africana $\left(n^{\circ} 164\right)$; 18 , Combretaceae $\left(n^{\circ} 135\right) ; 19$, Burkea africana $\left(n^{\circ} 167\right)$ 
échantillons qui proviennent d'une même localité, par exemple Parakou, on peut penser que les abeilles disposaient d'une quantité variable de plantes fleuries aux alentours de leurs ruchers assez éloignés les uns des autres, ou encore que la qualité des productions offertes par ces fleurs plus ou moins nectarifères les a incitées à récolter plus ou moins de nectar contaminé de pollens. Dans le cas où certains ruchers auraient été proches les uns des autres, la variation du nombre de pollens dans les différents échantillons peut aussi s'expliquer par la compétition entre les abeilles (Louveaux, 1980) qui ne peuvent utiliser, toutes à la fois, les plantes les plus fleuries proches de leur rucher.

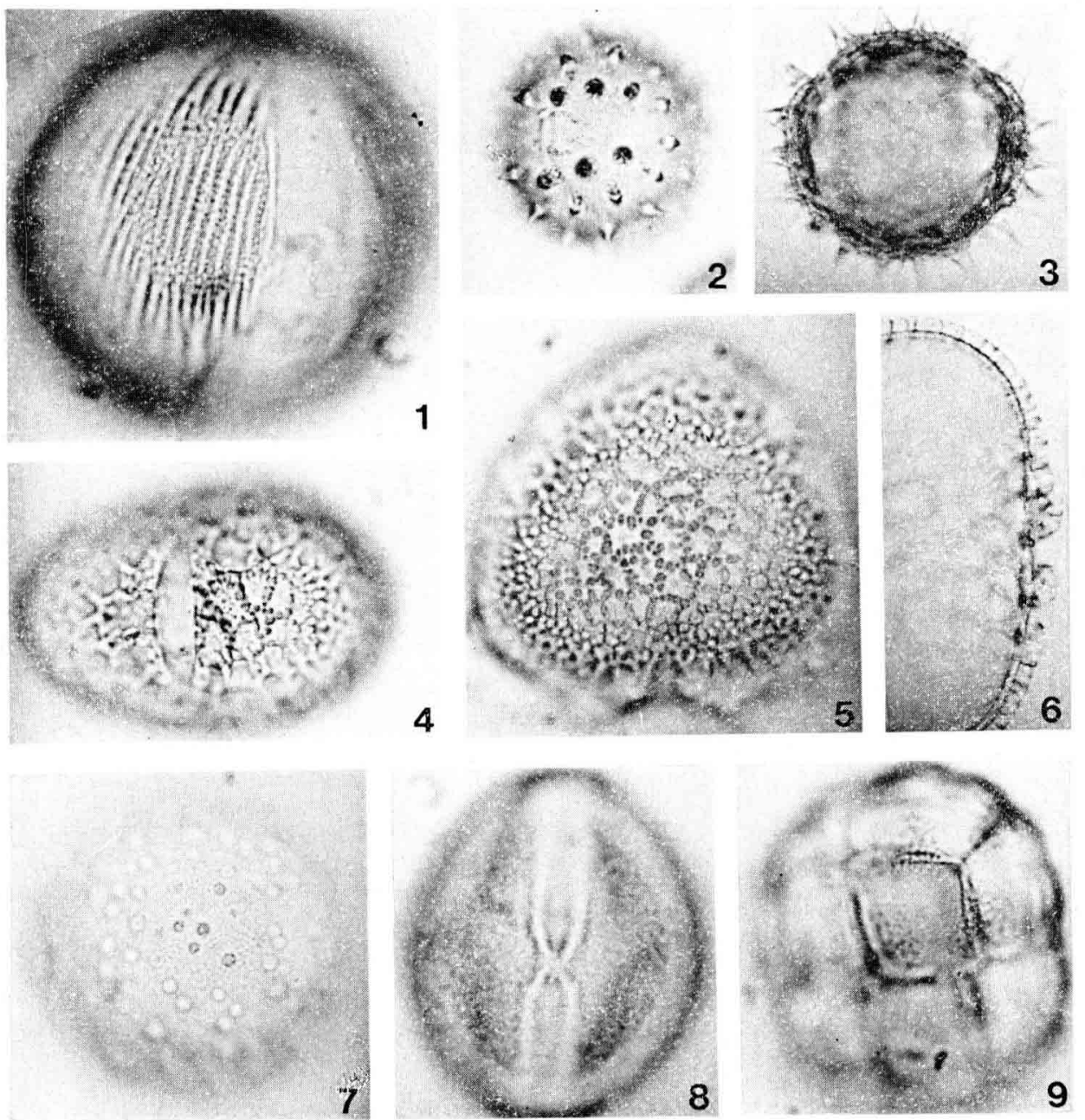

FIG. 3. - $(G r \times 1000)$. - 1 , Isoberlinia doka $\left(n^{\circ} 167\right) ; 2-3$, cf. Bidens sp. $\left(n^{\circ} 167\right)$;

4-6, Bombax of. costatum ( $\left.n^{\prime \prime} 164\right) ; 7$, Borassus $c f$. aethiopicum ( $\left.n^{\circ} 135\right) ; 8$, Cassia sp. $\left(n^{\circ} 171\right)$;

9, Acacia sp. ( $n^{\circ}$ 167) 


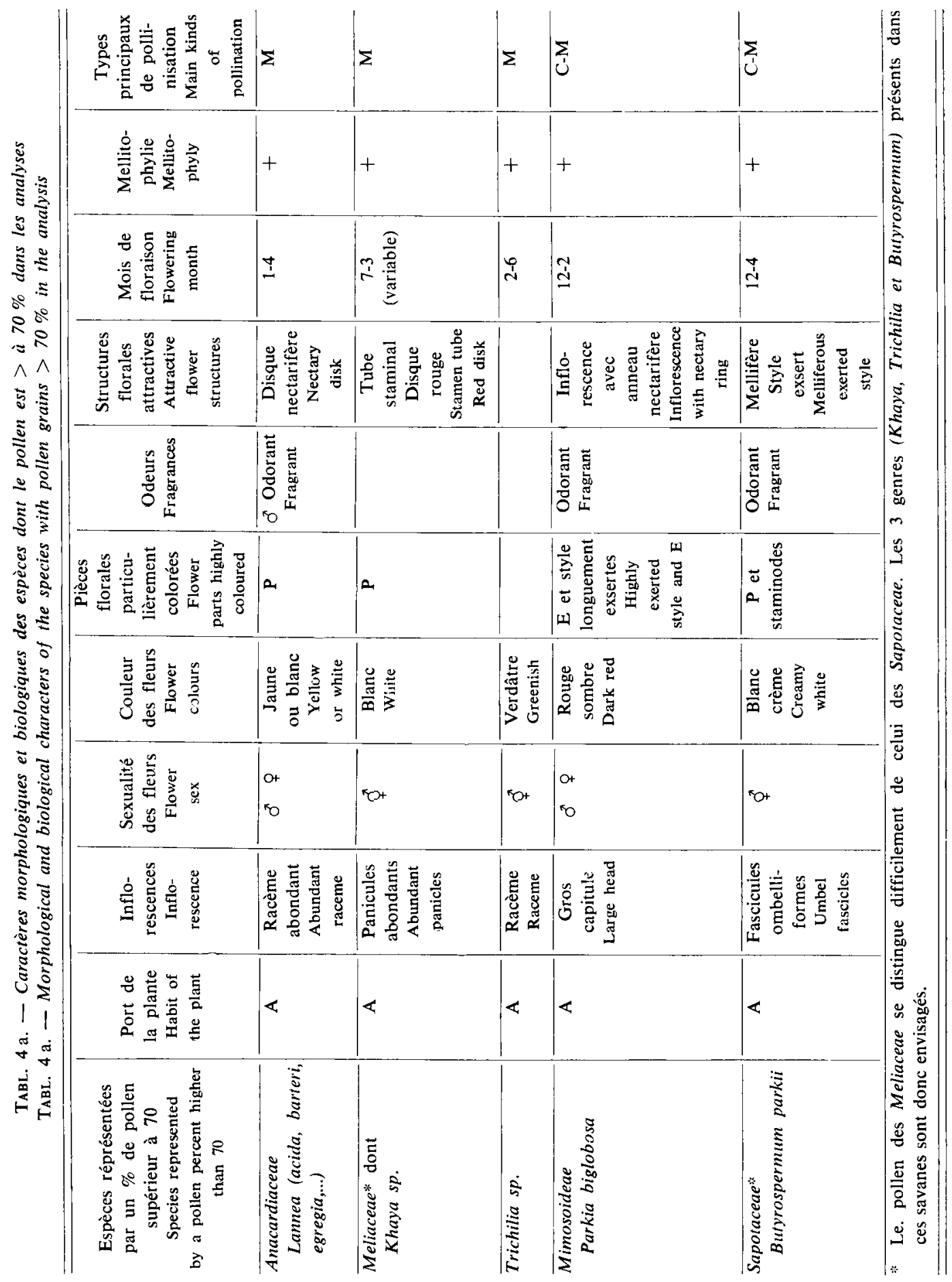




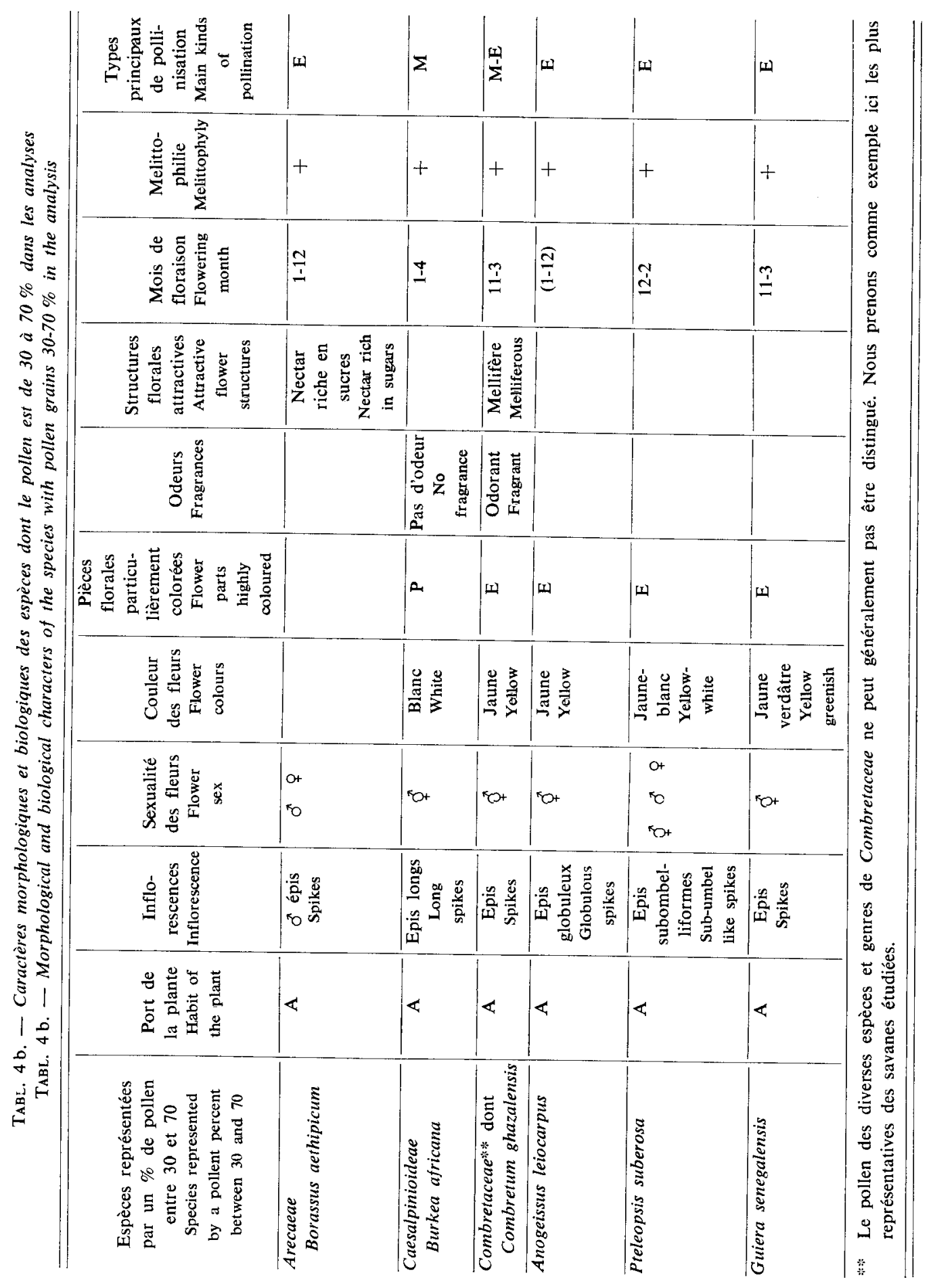




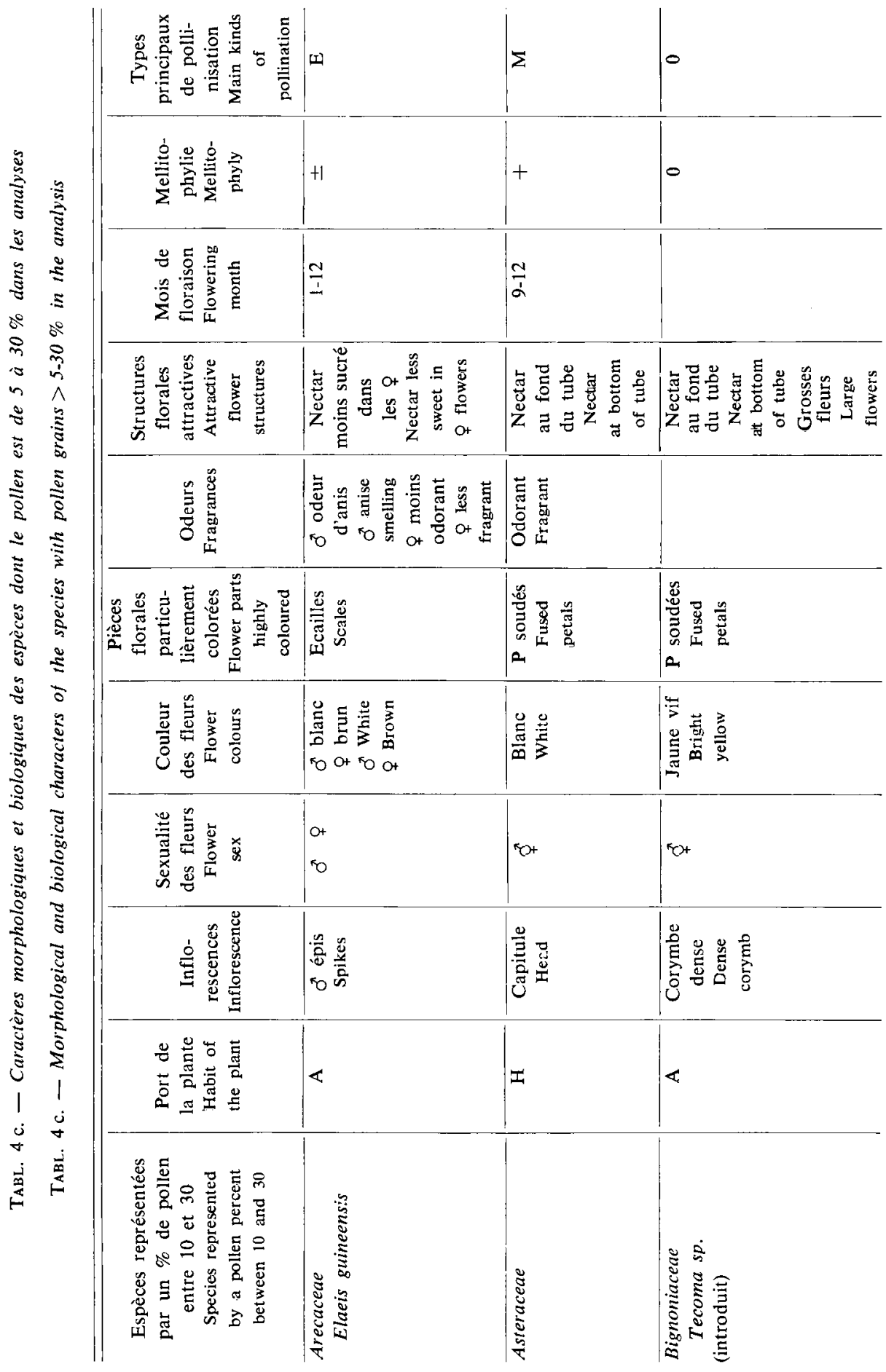




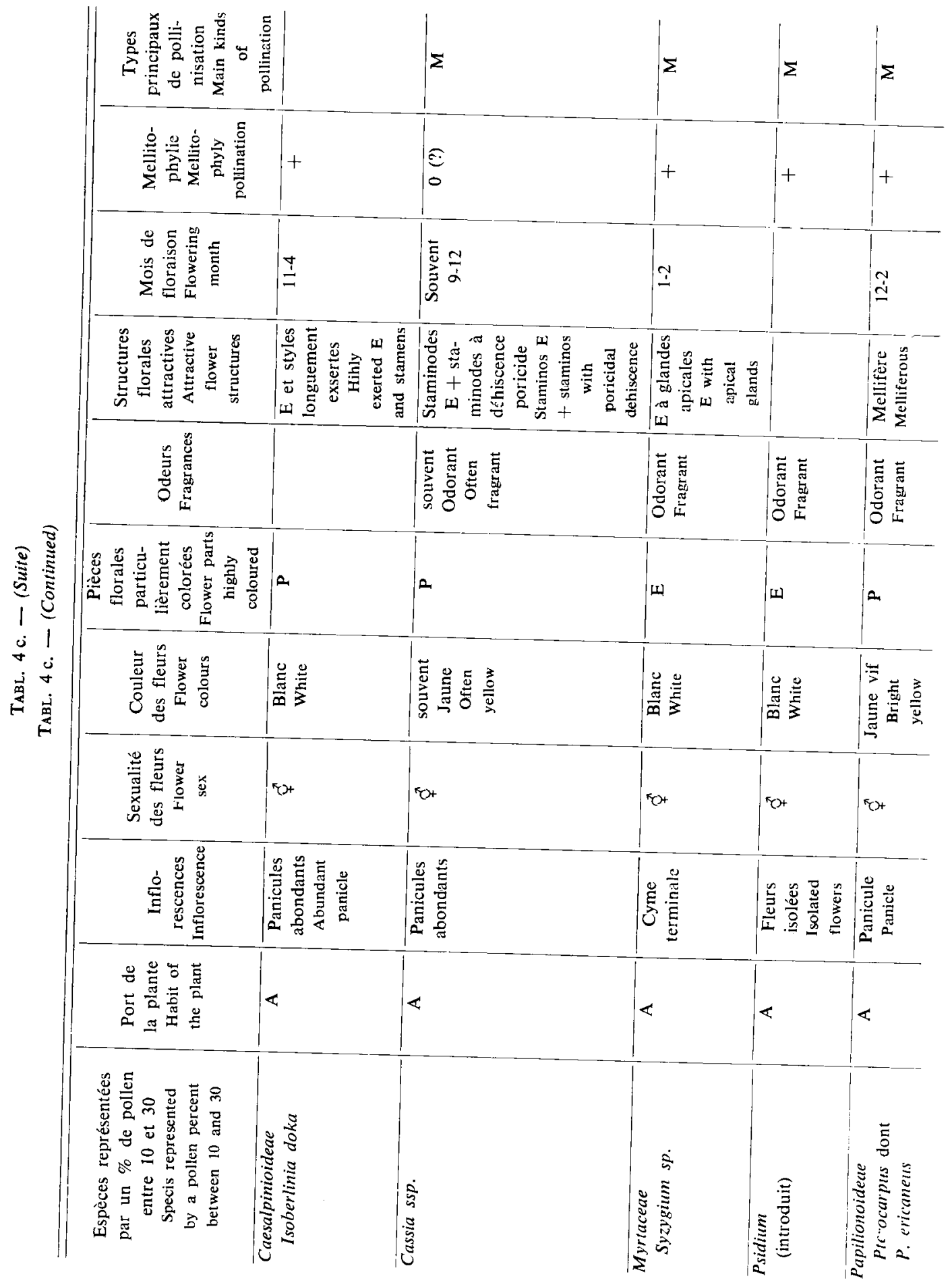




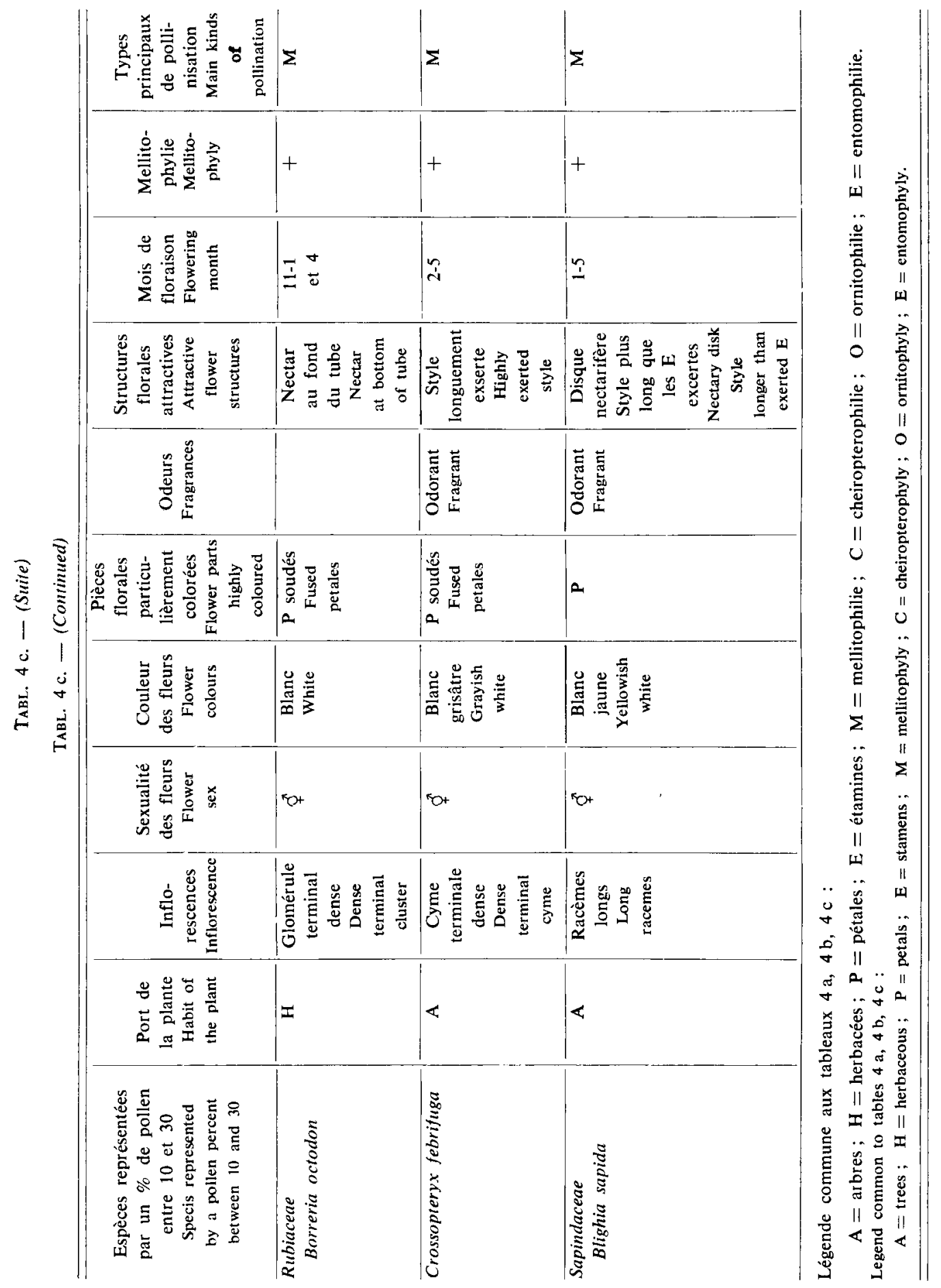


Malgré l'incidence du mode de comptage des pollens, le total des pollens comptés pour chaque échantillon permet de montrer globalement que la stratégie de butinage des abeilles peut dépendre de plusieurs facteurs complémentaires : production de fleurs dans l'environnement, qualité de leur production pollinique et nectarifère, compétition entre les abeilles. Cette première constatation générale paraît pouvoir également être soutenue par le caractère très sélectif des plantes récoltées et trouvées dans chaque échantillon (tabl. 2). En effet, si l'on observe les résultats concernant l'ensemble des échantillons de miels d'A pis, on constate que les espèces végétales dont le pollen est dominant dans les analyses $(>5 \%)$ représentent un nombre relativement restreint par rapport au nombre de genres identifiés dans les analyses (36) et surtout par rapport à l'ensemble des plantes disponibles en savane à cette époque de l'année (Aubreviri.li, 1950, 1959: Hutchinson et Dalziel, 1972 ; Ern, 1984). Au Togo et au Bénin, Apis mellifert et Hyptotrigona sp. n'échappent donc pas à la règle, commune aux espèces eurspéennes, selon laquelle peu d'espèces végétales sont visitées par rapport au nombre de celles qui sont fleuries à une même époque de l'année (LeppIK, 1957: Louveaux, 1958 ; Maclor, 1974 ; Waddington et Holdin, 1977).

D'autre part, si l'on compare les échantillons provenant des ruches à cadres avec les miels d'extraction traditionnelle, on constate qu'ils ont tous une composition floristique très proche avec une diversité pollinique comparable. Tous les pollens proviennent de fleurs nectarifères, à l'exception de ceux de Cassia qui sont strictement réservés aux miels d'extraction traditionnelle. Les traces infimes que l'on retrouvent dans un seul miel provenant de ruchers modernes (161) peuvent s'expliquer par l'ouverture des cellules à pollens au moment de la centrifugation des rayons. Cette homogénéité qualitative des deux types de miels, montre donc que les abeilles sélectionnent en priorité les fleurs en fonction de leur production nectarifère.

Dans la discussion, nous allons tenter d'anaiyser plus en détail les raisons bio-écologiques de la stratégie des deux genres d'abeilles : Apis et Hypotrigona et d'examiner les différences qui apparaissent entre elles.

\section{DISCUSSION}

La flore du Togo et du Bénin est assez bien connue des botanistes, en particulier par les travaux de Chevalier, 1920; Aubrevillli:, 1937, 1938. 1950, 1959 ; Hutchinson et Dalziel, 1954-1972 ; Morel., 1983, Ern, 1985). La composition floristique est comparab!e pour les deux pays dont la plus grande partie est caractérisée par les formations de savanes boisées guinéennes, à aspect de forêts claires, avec des galeries forestières pouvant s'élargir à certains endroits en bande de forêt de type équatorial. Cet aspect très boisé est très largement 
dû à la présence de peuplements d'Isoberlinia (Caesalpinioideae) qui dominent la région centrale des deux pays dont la pluviométrie annuelle est d'au moins $1300 \mathrm{~mm}$ et où ont été récoltés la plupart des miels analysés.

Quelques autres espèces sociales arborescentes sont caractéristiques de ces savanes, telles qu'Anogeissus schimperi, Daniellia oliveri, mais surtout Uapaca somon (Euphorbiaceae) disséminé par taches dans toute l'aire d'Isoberlinia et particulièrement abondante dans la région de Sokodé. Fréquent dans les galeries forestières, Khaya senegalensis (Meliaceae) domine la savane en individus isolés, dans la région de Parakou. D'autres essences arborescentes sont également très significatives dans cette savane boisée, mais disséminées un peu partout : Pterocarpus erinaceus (Papilionoideae), Afzelia africana (Caesalpinioideae), Ceiba pentandra (Bombacaceae), Butyrospermum parkii (Sapotaceae) ou encore Parkia biglobosa (Mimosoideae) typique des savanes soudaniennes plus sèches et surtout très commun dans les anciens terrains de culture, Lophira alata (Ochnaceae), Monotes kerstingii (Dipterocarpaceae), Lannea spp. (Anacardiaceae), etc.

Plusieurs grands arbres, relativement abondants dans toutes les savanes où ont été récoltés les échantillons de miel, ont pu être identifiés : Parkia biglobosa, Butyrospermum parkii, Pterocarpus erinaceus, Afzelia africana ; moins fréquents : Pterocarpus sp., Tamarindus indica, Prosopis africana, Entada africana, Daniellia oliveri, Burkea africana, Blighia sapida. Parmi les arbustes, on a pu noter la présence de plusieurs espèces d'Acacia, de Combretaceae, Piliostigma sp., Annona senegalensis et Balanites aegyptiaca. La strate herbacée est dominée par Bidens (Asteraceae) et Stachytarpheta (Verbenaceae).

Dans la région de Parakou (Bénin Centre), les floraisons les plus remarquables correspondaient à Daniellia oliveri, Isoberlinia doka, Lannea sp., Pterocarpus erinaceus, $P$. santalinoides et Syzygium guineense.

Les régions de latitudes plus élevées où ont été faites certaines récoltes de miels (Boukoumbé) ont une végétation de savanes souvent très dégradée à la suite d'un fort accroissement démographique.

Comparativement à la richesse relative de cette flore dont nous n'avons mentionné que les espèces dominantes, le spectre pollinique des miels étudiés montre une certaine pauvreté. On y retrouve plusieurs espèces caractéristiques de ces régions, mais d'autres en sont totalement absentes, telles qu'lsoberlinia, Uapaca somon, Anogeissus schimperi, Monotes kerstingii, Lophira alata, Daniellia oliveri, Piliostigma dans la strate arborée, ainsi que Bidens et Stachytarpheta dans la strate herbacée. La flore mellifère du Togo et du Bénin est donc typiquement représentative de ces régions, mais très nettement appauvrie. Cette constatation générale rejoint les remarques que nous avons faites sur les écarts qui existent dans la richesse pollinique des différents échantillons et trouve sans doute en partie son explication dans le champ d'action limité des abeilles. 
Cependant cela ne peut suffire à expliquer l'attirance des abeilles pour certaines essences particulières.

\section{Apis mellifera}

Quatre familles sont en effet particulièrement recherchées par Apis $(\because 70 \%$ de pollens par rapport à la somme totale des pollens comptés); les Sapotaceae (Butyrospermum parkii), les Meliaceae (") (2 espèces de Khaya et 2 espèces de Trichilia), les Mimosoideae (Parkia biglobosa) et les Anacardiaceae (Lannea), la plupart de ces espèces étant connues comme mellifères (tableau $4 \mathrm{a}$ ).

La sélectivité d'Apis mellifera pour ces plantes qui paraissent exercer sur elle une attractivité toute particulière ne peut s'expliquer sans faire intervenir plusieurs facteurs puisque les très forts pourcentages polliniques ne se retrouvent pas systématiquement dans tous les échantillons. En premier lieu, ce sont des espèces dont la période de floraison correspond aux mois précédant les récoltes de miels. D'autre part, ces espèces sont souvent anthrophiles, particulièrement abondantes près des villages, sur les terrains de culture (Parkia biglobosa), pouvant constituer des sortes de vergers (Butyrospermum parkii, Khaya senegalensis). Elles peuvent donc être privilégiées dans l'environnement des nids d'abeilles et des ruchers, au moins dans l'apiculture indigène. Leur morphologie est également caractéristique de l'attractivité constatée chez les abeilles : de larges cimes portant des inflorescences très denses, en ombelles, épis ou gros capitules très fleuris à l'extrémité des rameaux défeuillés (tabl. 4a). Enfin, ces espèces sont connues pour leur production abondante de nectar parfumé (AUBREvill.e, 1950) et riche en saccharose (FAegri et VAN der Piji, 1979 ; Baker et Bakiz, 1983).

Cependant, le cas de Parkia et des Sapotaceae pose un problème puisque ce sont des plantes décrites depuis longtemps comme cheiroptérophiles (VAN DER Pijl, 1937 ; Vogel, 1954, 1969 ; AYENSU, 1974). Les chauve-souris sont attirées par la forte odeur du nectar secrété en grande quantité à la base du capitule, au niveau des fleurs mâles ou stériles de Parkia (BAKEr et Harris, 1957 ; VoGEL, 1969). Pourtant, Aubreville dès 1950, indique que Butyrospermum est mellifère et, après les observations de BAKER et HARRIS (1957) certains auteurs (AYENSU, 1974 ; Hopkıns, 1983-1984) considèrent que les abeilles sont également pollinisatrices des fleurs de Parkia. D'autres observations, telles que celles de VogeL (1954, 1968), VAn Der PiJl (1937) et Carvalho (1960) montrent, au contraire, que si les abeilles visitent en grand nombre les fleurs de Parkia, il ne semble pas

(2) Malgré la très grande similitude des pollens de Sapotaceae et de Meliaceae pour lesquels il existe une série morphologique continue, il a été possible, dans certains cas, de distinguer les types extrêmes et de les identifier sans qu'il soit possible de les différencier systématiquement dans les comptages. 
possible qu'elles puissent en assurer la pollinisation. En effet, si les fleurs stériles de la couronne basale s'ouvrent au cours de l'après-midi, les fleurs hermaphrodites protandres ne s'ouvrent pas avant l'obscurité complète. Au début de la nuit, les étamines rouge sombre sont alors fertiles, puis il y a secrétion de nectar et enfin, au milieu de la nuit les styles s'allongent et les stigmates deviennent réceptifs, ce qui entraîne un changement de couleur de la fleur. Le lendemain matin, les fleurs ne sont plus fertiles, les stigmates n'étant plus réceptifs, mais les abeilles sont cependant attirées en grand nombre par les odeurs et le pollen encore abondant. Les abeilles ne seraient donc pas des agents pollinisateurs de Parkia, mais seulement de fortes consommatrices de pollen, comme on peut le voir dans les analyses de miels.

En outre, on remarque que, tous les échantillons qui contiennent du polien de Parkia (quelque soit le pourcentage), renferment une quantité importante de gomme ou mucilage, colorable au soudan III, ce qui indique la présence de lipides. Ces substances ne peuvent provenir du pollen lui-même puisqu'elles sont totalement absentes lorsqu'on traite des pelotes d'abeilles solitaires (LOBREAU-CALLEN, inéd.). Elles ne semblent pas davantage appartenir à un type de secrétions florales accessoires assurant une fixation supplémentaire du pollen sur les poils des animaux pollinisateurs, telles qu'elles sont décrites par VoGEL (1984) qui ne les mentionne nullement dans les fleurs de Parkia (1968). Le nectar de Parkia, laiteux et abondant (VAN DER PIJL, 1937 ; JAEGER, 1954) pourrait aussi être la source de ces substances. On sait, en effet, que de nombreuses fleurs de plantes cheiroptérophiles (Bignoniacées, par exemple), renferment des lipides qui donnent un aspect laiteux au nectar, ce qui pourrait laisser penser que le nectar de Parkia contient également des lipides. Mais on ne peut concevoir qu'un tel nectar, toujours très liquide, puisse donner, au cours des traitements acides que l'on a pratiqués, des gels comparables à ceux que l'on trouve en abondance dans les échantillons renfermant du pollen de Parkia.

En revanche, de nombreuses Mimosacées, dont Parkia, produisent sur leur tronc, des gommes ou résines riches en huiles essentielles, colorables au soudan III. Elles pourraient alors être utilisées par les abeilles qui recherchent et stockent la propolis (gomme, résine, huiles essentielles, etc.) pour entretenir leurs ruches. Comme dans la propolis récoltée par les abeilles en Europe, on trouve en effet, déposés à la surface de ces substances accompagnant le pollen de Parkia, un petit nombre de grains de pollens d'espèces variées. Ceci pourrait aussi expliquer la présence de pourcentages infimes de pollens de plantes anémogames, tels que Celtis ou Graminées, rencontrés dans nos échantillons de miels. D'autre part, l'absence totale de ces substances dans des miels de Haute-Volta contenant un faible pourcentage de pollens de Parkia (Lobreau-Callen, 1986), laisse également penser qu'elles n'appartiennent pas au nectar, mais à la propolis que les abeilles ne récolteraient qu'en fonction de leurs besoins. 
Sur les plantes cheiroptérophiles, les abeilles ne se comporteraient donc pas en pollinisatrices, mais en fortes consommatrices de nectar, parce qu'il est abondant et riche en saccharose (Pettet, 1977 ; Baker et BAKER, 1975, 1983) et de pollen pour ses réserves protéiniques et lipidiques, substances qui leur assurent, ainsi qu'aux larves, une importante source énergétique avec des dépenses caloriques restreintes. Dans ce cas, l'équilibre énergétique démontré par HEINRICH et RAVEN (1972) est très facilement assuré. De plus, selon leurs besoins et, sans doute à des époques précises, les abeilles recherchent plus spécialement la propolis pour leurs ruches, comme on le voit sur le Parkia.

Moins attractives que ces quatre familles que nous venons d'analyser les Leguminosae-Caesalpinioideae (Burkea, Cassia), les Combretaceae et une Arecaceae (Borassus aethiopicum) sont cependant encore abondamment butinées par les Apis (30 à $70 \%$ de pollens). Si elles paraissent très bien adaptées à la pollinisation par les abeilles, soit par leur abondante floraison, leur morphologie florale (tabl. $4 \mathrm{~b}$ ) avec en particulier de nombreuses étamines ou des staminodes produisant dans certains cas (Cassia) un petit pollen stérile récolté par les abeilles (MichenER, 1964 ; Buchman et al., 1981 ; Buchman, 1983 ; Macior, 1971, $1974 \mathrm{a}, \mathrm{b}$; Baker et Hurd, 1968 ; Faegri et VAN Der PiJl, 1979), soit par la composition chimique de leur nectar abondant, odorant (AUBrEville, 1950) ; et riche en saccharose (BAKER et BAKER, 1975, 1983 ; SoutHwick et al., 1981), elles paraissent être légèrement délaissées au bénéfice de plantes plus attractives et plus riches en ressources énergétiques.

Nettement moins recherchés, Blighia sapida (Sapindaceae), Bidens (Asteraceae), Borreria octodon, Crossopteryx febrifuga (Rubiaceae), Psidium, Syzygium (Myrtaceae), Isoberlinia doka (Caesalpinioideae), Pterocarpus spp. (Papilionoideae) Elaeis guineensis (Arecaceae) sont représentées par des pourcentages compris entre 10 et $30 \%$ (tabl. 4c). L'odeur particulière de leur nectar souvent assez forte (Blighia, odeur d'anis chez Elaeis,...), la morphologie de la fleur plutôt adaptée à la pollinisation par les Coléoptères (Elaeis guineensis : DESMIER DE Chenon, 1981 ; Pouvreau, 1984) ou plus simplement la distance de ces arbres par rapport aux nids sont autant de raisons qui peuvent être invoquées pour expliquer leur moindre attraction sur les abeilles.

L'absence totale de pollens de Daniellia oliveri dans les analyses paraît importante, puisqu'il s'agit d'une espèce commune des savanes boisées soudaniennes et soudano-guinéennes surtout dans les pays habités et cultivés et en floraison à cette époque. La préférence qu'elle manifeste pour les terrains humides l'éloignait peut-être davantage des nids à moins qu'il ne s'agisse d'un choix délibéré de la part des abeilles.

Deux seules herbacées sont présentes dans quelques-uns des échantillons analysés : Bidens sp. (Asteraceae) et Borreria (Rubiaceae) toujours avec un 
pourcentage inférieur à $25 \%$. La période de floraison des herbacées est pratiquement terminée en décembre, alors qu'elle commence pour un grand nombre d'arbres qui fieurissent pendant toute la saison sèche (novembre-avrii). Ce décalage dans la floraison des différentes strates est susceptible d'expliquer la présence presque exclusive de ligneux dans les analyses effectuées qui ne correspondraient donc qu'au butinage des abeilles pendant les 4-5 mois de période sèche précédant la récolte des miels.

\section{Hypotrigona sp.}

L'analyse de deux échantillons de miels récupérés dans deux nids d'Hypotrigona sp., abeilles sans dard, de petites dimensions, et provenant des régions centrales du Bénin et du Togo, peut aider à mieux comprendre le comportement de butinage d'Apis mellifera, sa stratégie, son choix de pollens, les facteurs déterminant en partie l'ampleur du spectre pollinique trouvé dans les miels.

Le tableau récapitulatif des résultats (tabl. 2) montre déjà, que durant la même saison sèche, les Hypotrigones visitent essentiellement des plantes différentes de celles butinées par Apis : au Bénin, elles préfèrent Borassus aethiopicum $(47,7 \%)$ et Syzygium (Myrtaceae) (34,3\%) et au Togo, les Sapindaceae telles Blighia sapida $(60,0 \%)$.

Apis et Hypotrigona ne se retrouvent parfois que sur les fleurs des Leguminosae (Parkia, Burkea) et des Sapotaceae (Butyrospermum), groupées en grosses inflorescences particulièrement nombreuses et où les étamines sont abondantes. Toutefois les pourcentages des récoltes en pollen de ces différents taxons botaniques par Apis et Hypotrigona sont très différents. Il est impossible pour le moment de savoir s'il y a ou non compétition entre ces deux groupes d'abeilles. Seules, davantage d'observations sur le terrain nous permettraient de comprendre comment s'effectue ce chevauchement. Existe-t-il une agressivité entre les butineuses des différentes espèces d'abeilles? s'ignorent-elles? ou bien encore ont-elles adopté des heures différentes de travail comme elles le font avec les chauves-souris, les oiseaux, les papillons (lorsque les sources de récoltes sont communes)? Ces différences dans le butinage sont-elles fonction de la composition chimique des nectars, soit de diverses espèces botaniques, comme cela a été montré par BaKer $(1977,1978)$, BAKER et BAKer (1973 à 1983) et Southwick et al. (1983), soit dans les variations des concentrations des différentes substances qui le composent ou de sa concentration globale au cours de la journée (WiLlmer, 1980), du développement des étamines, du degré de maturité du pollen ou de la croissance du style et des stigmates?

L'analyse comparée des miels récoltés par Apis et Hypotrigona montre seulement qu'Apis semble préférer avant tout des fleurs aux nectars très abondants, riches en sacccharose et aux pollens avec une abondante couche de 
pollencoat lipidique répartie à la surface de l'exine. Les Hypotrigones fréquentent également ces fleurs, mais recherchent avant tout des plantes dont on sait qu'elles renferment des substances à odeurs fortes, peut-être retrouvées dans les nectars (Blighia, Syzygium), certaines de ces substances (alcaloïdes, lipides, acides aminés, phéno's) étant répulsives pour certains insectes et attractives pour d'autres, surtout lorsqu'elles sont présentes dans les nectars (BAKER et BAKER, 1975, 1983).

L'appétance des Hypotrigones (s.l.) pour les nectars at les pollens de certaines Sapindaceae a déjà été mise en évidence dans les régions néotropicales (BAWA, 1977 ; Iwama et Mehlem, 1980 ; Sommeijer et al., 1983). L'analyse des miels du Togo et du Bénin paraît élargir cette observation à certaines espèces africaines (Paullinia, Blighia sapida). Les trigones seraient-elles plus particulièrement attirées par des substances communes à plusieurs Sapindaceae dont on sait que le nectar de certaines d'entre elles contient des alcaloïdes ou autres substances toxiques (Wulfrath et Speck ; BaKer et BaKer, 1975)?

\section{CONCLUSION}

L'analyse des récoltes faites par les insectes est un aspect original de l'étude des phénomènes biologiques et en particulier des interractions plantes/insectes, mais elle a évidemment ses limites dans le domaine de la pollinisation qui doit faire appel à d'autres techniques, en particulier sur le terrain. Néanmoins, cette analyse de miels apporte certains renseignements sur la stratégie des abeilles et cela, en dépit même du faible échantillonnage portant sur du matériel dont les conditions de récoltes ne sont pas toujours suffisamment précises.

La première constatation qui s'impose est l'intérêt que manifestent de façon privilégiée les abeilles pour certaines espèces arborescentes, quelles soient ou non leur pollinisateur. $\mathrm{Si}$, manifestement ces espèces appartiennent à la strate la plus fleurie à l'époque des récoltes analysées, elles ne sont pas les seules et indiquent alors un choix relativement restreint de la part des insectes. Les plantes les plus visitées sont particulièrement fréquentes dans les régions de culture où sont installés les ruchers, non loin des villages, ce qui traduit de la part des abeilles la recherche d'une dépense énergétique minimale. En outre, les fleurs de ces espèces favorisées, généralement de teinte claire (blanche ou jaunâtre), émettent des odeurs attractives et secrètent un nectar abondant et riche en substances recherchées par les abeilles. Sans observation directe, il est difficile de préjuger du rôle pollinisateur qu'elles peuvent jouer sur toutes ces plantes, mais nous avons vu que, dans certains cas où leur action est largement associée à celle des chauvessouris, il est vraisemblable qu'elles soient seulement des utilisatrices et qu'elles n'exercent aucune action dans la pollinisation. 


\title{
REMERCIEMENTS
}

Le séjour de R. Darchen au Bénin et au Togo a été effectué grâce au Fond Européen de Développement qui a entièrement financé sa mission. MM. R. Letouzey et J.F. Vitliters (Muséum) nous ont fait part de leurs observations sur le terrain. La majeure partie des techniques palynologiques a été assurée par N. D'Amico (E.P.H.E.), les tirages photographiques par T. Derorn (E.P.H.E.), la carte géographique par le bureau de dessin de I'I.N.R.A., et la dactylographie par J. Ramfau (C.N.R.S.) et F. Joligeon (Muséum).

\section{ZUSAMMENFASSUNG}

\author{
BEITRAG DER POLLENANALYSE ZUR KENNTNIS DER BEZIEHUNGEN \\ ZWISCHEN BIENEN UND PFLANZEN \\ IN DEN WALDSAVANNEN VON TOGO UND BENIN
}

13 Honigproben aus den Savannen vom Zentrum und Norden von Togo und Benin wurden pollenanalytisch untersucht. In diesem Gebiet wird die Bienenzucht sowohl nach traditionellen wie nach modernen Methoden betrieben. Zwei Proben stammen von natürlichen Nestern von Hypotrigona. Diese Analysen haben folgendes ergeben :

- Eine sehr variable Breite des Pollenspektrum in Bezug auf die Quantität der Pollenkörner in den verschiedenen Proben. Diese Unterschiede lassen sich durch die angewandte Zählmethode erklären, durch den hohen Prozentsatz sehr großer Polyaden (mit 32 Körnern) in einigen Proben sowie durch die Ergiebigkeit der Blüten rund um die Bienenvölker, die sich in cincm beschränkten Aktionsradius der Bienen ausdrückt.

- Eine beschränkte Bienenflora im Verhältnis zur Gesamtheit der in der Umgebung blühenden Pflanzen, mit einigen Arten, die für Afrika zum ersten Mal festgestellt wurden.

- Eine sehr charakteristische Auslese der Pflanzen, die in großer Zahl beflogen wurden (zwei oder drei per Probe), und zwar wegen ihrer Blütezeit, ihres Charakters als Kulturpflanze, ihrer attraktiven Morphologie und ihrer Produktion von Nektar mit hohem Saccharosegehalt. Diese Auslese ist für Apis und Hypotrigona verschieden.

- Eine Bevorzugung von baumartigen Trachtpflanzen zur Trockenzeit.

- Eine Attraktivität der Bienen durch gewisse cheiropterophile (Fledermaus-bestäubte) Arten (Parkia), auf welchen sie sich als Sammler von Pollen und zusätzlichen Substanzen für den Bedarf im Bienenstock (z.B. Harz) betätigen, und nicht als Bestäuber.

\section{SUMMARY}

\author{
CONTRIBUTION OF PALYNOLOGY \\ TO THE KNOWLEDGE OF BEE-PLANT RELATIONSHIPS \\ IN THE FOREST SAVANNAS OF TOGO AND BENIN
}

Pollen analysis was conducted on 13 samples of honey collected in the savannas of Central and Northern Togo and the Benin Republic, where beekeeping is practised according to both traditional and modern methods. Two of the samples are from honey combs of Iyporrigona sp., collected in the wild. This analysis shows :

- a highly variable breadth of the pollen spectrum as to the quantity of pollen grains within the different samples. These differences were due to the method chosen for counting as well as 
to the high percentage of very large polyads (32 pollen grains) found in some samples. The yield of flowers in the neighborhood of the bee hives revealed a restricted area of operation of the bees concerned;

- a restricted melliferous flora in relation to the flowering representatives of the vegetation of the environment, with some species newly recorded for Africa;

- a very characteristic selection of plants visited in great number ( 2 or 3 per sample) because of their flowering periods, their synanthropic character, their attractiveness from the morphological point of view of the insect and, finally, their abundant production of nectar rich in saccharose. The selection was different for Apis and Hypotrigona;

- the preference given by bees to flowering trees during the dry season;

- an attractiveness to bees of some cheiropterophilous (batpollinated) species (e.g. Parkia), which they visit not only as pollinating agents, but in order to gather from them pollen and other substances for various uses in their hive.

\section{REFÉRENCES BIBLIOGRAPHIQUES}

Aubreville A., 1937. - Les forêts du Togo et du Dahomey. Bull. du Com. Hist. et Scien. de l'A.O.F., 20 (1-2), 1-112.

Aubreville A., 1938. - La forêt coloniale. Acad. Sci. Coloniales, Annales, 9, 244 p., 18 pl.

Aubreville A., 1950. - Flore forestière soudano-guinéenne. A.O.F.-Cammeroun-A.E.F. Soc. d'Ed. Géographique Maritimes et Coloniales, Paris, $521 \mathrm{p}$.

Aubreville A., 1959. - Flore forestière de Côte d'Ivoire. C.T.F.T., Nogent,-sur-Marne, 3 volumes. Ayensu E.A., 1974. - Plant and bat interaction in West Africa. Ann. Miss. Bot. Gard., 61, $702-727$. Baker H.G., 1977. - Non-Sugar chemical constituents of nectar. Apidologie, 8, 349-356.

BAKER H.G., 1978. - Chemical aspects of the pollination biology of woody plants in the tropics. In : P.B. Tomlinson \& M.H. Zimmerman eds., Tropical Trees as living systems, Cambridge University Press, Cambridge, 64-68.

Baker H.G., Baker I., 1973. - Amino acids in nectar and their evolutionary significance. Nattre, Lond., 241, 543-545.

BAKer H.G., BaKer I., 1975. - Studies of nectar constitution and pollinator-plant coevolution. In : L.E. Gilbert \& P.H. Raven eds., Coevolution of animals and plants, University of Texas Press, Austin, 100-140.

BaKer H.G., BaKer I., 1983. - A brief historical review of the chemistry of floral nectar. $I n$ : B. Bentley \& T. Elias eds., The biology of nectaries, Columbia University Press, New York, 126-152.

Baker H.G., Harris B.I., 1957. - The pollinisation of Parkia by bats and its attendant evolutionaty problems. Evolution, 11, 449-460.

Baker H.G., Hurd P.H., 1968. - Intrafloral ecology. Ann. Rev. Entomol, 13, 385-414.

BAWA K.S., 1977. - Reproductive biology of Cupania guatemalensis Radlk. (Sapindaceae). Evolution, 31, 52-63.

Buchman S.L., 1983. - Buzz pollination in angiosperm. In : C.E. Jones \& R.J. Little eds., Handbook of Experimental Pollination Biology, 73-113.

Buchman S.L., Buchman M.D., 1981. - Anthecology of Mouriri myrtilloides (Melastomataceae : Memecylaea), an oil flower of Panama. Biotropica, 13 (2), 7-24. Supplement on reproductive botany. 
Brunel J.F., Hiepko P., Scholz H., 1984. - Flore analytique du Togo, Phanérogames. Englera, 4, 1-751.

Carvalho C.T. de, 1960. - Das visitas de Morcegos às Flores. Anais Acad. Bras. Ciencias, 32 (3-4), 359-377.

Chevalier A., 1920. - Exploration botanique de l'A.O. Française. T. 1, Enumération des plantes, Lechevallier ed., Paris, 798 p.

Crane E., 1973. - Honey sources of some tropical and subtropical countries. Bee Wld., 54 (4), $177-186$.

Crane E., Walker P., Day R., 1984. - Directory of important World honey Soturces. IBRA ed., I.ondon.

Desmier de Chenon R., 1981. - Entomophil pollination of oil palm in West Africa. Preliminary Research, Intern. Oil Palm Conference, Kuala-Lumpur, Malaysia, 219-319.

ERN H., 1984. - Les divisions écologiques du Togo. In : Flore analytique du Togo. Phanérogames, Brunel J.F., Hiepko P., Scholz H. eds. Englera, 4, 9-18.

Faegri K., Van der PIJl L., 1979. — The principles of Pollination ecology. 3" ed., Pergamon Press, Oxford, New York, Toronton, Sydney, Paris, Frankfurt, 295 p.

Gadis C., 1980. - Les plantes utilisées par les abeilles au Tchad méridional. Apidologie, 11 (2), 217-254.

Heinrich B., Raven P.H., 1972. - Energetics and pollination ecology. Science, 76, 597-602.

Hopkins H.C., 1983. - The taxonomy, reproductive biology and economic potential of Parkia (Leguminosae : Mimosoideae) in Africa and Madagascar, Bot. J. Linn. Soc., 87, 135-167.

Hopkins H.C., 1984. - Floral Biology and pollination ecology of the neotropical Species of Parkia. Journ. Ecol., 72, 1-23.

Hutchinson J., Dalziel J.M., 1954-1972. - The Flora of West Tropical Africa, 3 vol.

Iwama S., MELhem T.S., 1979. - The pollen spectrum of the honey of Tetragonisca angustula Latreille (Apidae, Melinoninae). Apidologie, 10 (3), 275-295.

JAEger P., 1954. - Les aspects actuels du problème de cheiroptérogamie. Bull. Inst. Fr. Afr. Noire, 16, 796-821.

LEPPIK E.E., 1957. - Evolutionary relationship between entomophilous plants and anthomophilous insects. Evolution, 11, 466-481.

LieuX M., 1980. - Acetolysis applied to microscopical honey analysis. Grana, 19, 57-61.

Lobreau-Callen D, 1985. - Structure exinique du pollen collecté par les Anthophores du Sénégal. Bull. Sci. Géol., Strasbourg, 38 (1), 99-106.

Lobreau-Callen D., 1986. - Comportement d'Apis mellifera var. adansonii dans deux milieux différents de savane arborée ouest-africaine. Actes Coll. Insectes sociaux, 3, 61-71.

Lobreau-Callen D., Callen G., 1983. - Quelle est la composition pollinique d'un miel exotique ? I. Bull. Soc. Versail. Sci. Nat., sér. 4, 9 (4), 70-85 (1982); II, 10 (1), 1-41 (1983).

Lobreau-Callen D., Coutin R., 1984. - Pollens et Apoides du Sénégal : pollinisation et comportement. V Symp. Internat. Pollinisation, Versailles, 27-30 sept. 1983. Les Colloques de l'I.N.R.A., 21, 267-273.

Lobreau-Callen D., Coutin R. (sous presse). - Ressources exploitées par quelques Apoïdes des zones cultivées en savane arborée sénégalaise durant la saison des pluies. Agronomie.

Louveaux J., 1958. - Recherches sur la récolte du pollen par les abeilles (Apis mellifera L.). Thèse Fac. Sc. Univ. Paris, I.N.R.A., 206 p.

Louveaux J., 1980. — Les abeilles et leur élevage. Nouvelle Encyclopédie des Connaissances agricoles. Hachette éd., 235 p. 
Louveaux J., Maurizio A., Vorwohl. G., 1970. - Commission internationale de Botanique apicole de 1'I.U.B.S., les méthodes de mélisso-palynologie. Apidologie, 1 (2), 211-227.

L.ouveaux J., Maurizio A., Vorwohı. G., 1978. - Methods of melissopalynology. Bee World, 59 (4), 139-157.

MACIOR L.W., 1971. - Co-evolution of plants and animals-systematic insights from plant-insect interactions. Taxen, 20 (1), 17-28.

MACIOR L.W., 1974 a. - Pollinisation ecology of the front range of the Colorado rocky mountains. Melanderia, 15, 1-59.

Macior L.W., 1974 b. - Behavioral aspects of coadaptations between flowers and inseets pollinators. Ann. Miss. Bot. Gard., 61 (3), 760-769.

Maurizio A., 1968. - La récolte et l'emmagasinage du pollen par les abeilles. Traité de Biologie de l'Abeille, III, 168-173, Masson éd.

MCGregor S.E., 1976. - Insect pollination of cultivated crops plants. Agricular Handbook, 496.

Michener C.D., 1962. - An interesting methods of pollen collecting by bee from flowers with tubular anthers. Rev. Biol. Trop., 10 (2), 167-175.

Morel. J.C., 1983. - Les arbres et les arbustes des saranes outest-africaines. (Document pour l'étude de l'écologie des Glossines), ed. FAC, Paris, 2, 88.

Pettet A., 1977. - Seasonal changes in nectar-feeding by birds at Zaria. The Ibis, 119, $291-308$.

Pouvreau A., 1984. - Cultures tropicales oléagineuses, In : P. Pesson \& J. Louveaux, Pollinisation et productions v'égétales. I.N.R.A. ed., 331-348.

Schnell B., 1971. - Introduction à la phytogéographie des pays tropicalx. Paris, Gauthier-Villars cd., 2 vol.

Sмith F.G., 1957, - Bee Botany in East Africa. E. Afr. Agric. J., 23 (2), 119-126.

Simith F.G., 1960. - Beekeeping in the Tropics. Bristol, Longmans.

Sommlijer M.J., De Rooy G.A., Punr W., De Bruijn L.L.M., 1983. - A comparative study of foraging behavior and pollen resources of various stingless bees (Hym., Meliponinae) and honeybees (Hym., Apinae) in Trinidad, West-Indies. Apidologie, 14 (3), 205-224.

Southwick E.E., Loper G.M., SADwick S.E., 1981. - Nectar production, composition, energetics and pollinator attractiveness in spring flowers of Western New York. Amer. J. Bot., 68 (7), 994-1002.

Sowungr M.A., 1976. - The potential value of honey in paleoecology and archeology. Rev. Paleobotany Palynology, 21 (2), 171-185.

Van der Piji L., 1937. - Fledermäuse und Blumen. Flora, 31, 1-40.

Vergleron P., 1964. - Interprétation statistiques des résultats en matic̀re danalyse pollinique des miels. Ann. Abeille, 7 (4), 349-364.

Voges, S., 1954. - Blïtenbiologie Typen als Elemente der Sippengliederning, dargestellt an IIand aler Flora Sïdafrikas. Jena, $338 \mathrm{p}$.

Vogel, S., 1969. - Chiropterophilie in der neotropischen Flora. I-III. Flora, 157, 562-602; 158, $185-222,269-323$.

VoGel, S., 1984. - Blütenserkrete als akzessorischer Pollenkitt. Mitteilungs-Band Kurzfassungen der Beiträge. Bolanischer Tagung in Wien, 9-14 sept. 1984, DBG, p. 123.

Waddington K.D., Holden L.R., 1979. - Optimal foraging : on flower selection by bees. Am. Nat., 114 (2), 179-196.

Willmer P.G., 1980. — The effects of insect visitors on Nectar Constituents in Temperate Plants. Occologia (Ber1.), 47, 270-277.

Wulfrath A., Speck J.J., s. an, - La Flora melifera. Enciclopedia Apicola. Mexicanas ed., Mexico, 2d"ed., 28, 97. 